\title{
Diminished pulmonary function in pectus excavatum: from denying the problem to finding the mechanism
}

\author{
Robert E. Kelly Jr, Robert J. Obermeyer, Donald Nuss \\ Departments of Surgery and Pediatrics, Children's Hospital of The King's Daughters, Eastern Virginia Medical School, Norfolk, Virginia, USA \\ Correspondence to: Robert E. Kelly, Jr., MD, Surgeon-in-Chief. Children's Hospital of The King's Daughters, 601 Children's Lane, Ste. 5B, Norfolk, \\ Virginia, USA. Email: robert.kelly@chkd.org; robert.obermeyer@chkd.org.
}

Background: Recently, technical improvement in the ability to measure lung function and the severity of chest deformity have enabled progress in understanding the mechanism of limitations of lung function in pectus excavatum.

Methods: After establishing that most patients with pectus excavatum do have symptoms of exercise intolerance, easy fatigability, and shortness of breath with exertion, lung function has been evaluated by a variety of methods in different centers. Spirometry, plethysmography, exercise testing, oculo electronic plethysmography, and imaging methods have been used to assess lung function in pectus excavatum and its response to surgery.

Results: Not all patients with pectus excavatum have subnormal static pulmonary function testing; some have above-average values. However, in more than 1500 adult and pediatric surgical patients with anatomically severe pectus excavatum at a single center, the bell curve of FVC, FEV1, and FEF 2575 is shifted to significantly lower values in pectus excavatum. The curve is shifted to higher values after operation by approximately one standard deviation. Previous work has demonstrated that patients with more anatomically severe pectus excavatum are more likely to have diminished PFT's. A mechanism for this effect is seen by oculo electronic plethysmography, which demonstrates that the depressed portion of the chest does not move on respiration. After Nuss procedure, the chest wall motion used to create suction to draw air into the lungs is indistinguishable from that of persons with a normal chest, and the intrathoracic volume is markedly increased.

Conclusions: Pectus excavatum is accompanied in most patients by diminished static pulmonary function. Correction by Nuss procedure results in improvement in chest wall motion; this improvement in the thoracic bellows action is accompanied by improvement in pulmonary function testing.

Keywords: Pectus excavatum; pulmonary function; lung function; breathing mechanics; measurement of lung function

Submitted Sep 08, 2016. Accepted for publication Sep 25, 2016.

doi: 10.21037/acs.2016.09.09

View this article at: http://dx.doi.org/10.21037/acs.2016.09.09

\section{Introduction}

Lung function in pectus excavatum has been a controversial topic for decades. However, since the introduction of the Nuss procedure in 1997, there has been an explosion of investigations of the effects of the problem and its treatment. Now, there is clear evidence of physiologic effects of pectus excavatum on heart and lung function. The focus now needs to turn to the mechanism of these effects. The heart and lungs are a coupled system, so that effects on one system will likely affect the other. Dissecting these relationships, and correlating effects and severity, should take center stage.

The earliest reports of pectus excavatum described patients with trouble breathing. Clinicians noted exercise limitations that were improved by surgical treatment more 
than 70 years ago. When objective measurement of lung function was first employed, little improvement was seen with the crude methods of measurement then available. Recently, however, technical improvements in the ability to measure the severity of chest wall deformity and lung function have enabled clarification of the mechanism and effects of these phenomena.

This report seeks to review work on pulmonary function and pectus excavatum, and to update our institution's data previously reported data on this topic.

\section{Methods}

All consenting patients underwent pulmonary function testing by spirometry. The methods used are standard methods and have been described in previous work $[13,16]$. Testing was done in a variety of labs, not all of which are at our hospital. Collection and reporting of these data have been approved by the Eastern Virginia Medical School Institutional Review Board. Data were prospectively recorded in a database and retrospectively analyzed.

\section{Results}

Exercise intolerance, and in particular difficulty with breathing, have been linked with pectus excavatum from the beginning. The first known report of pectus excavatum was by Bauhinus, who described a patient from Andalusia, in what is now Spain, in the late 1500's. He concisely described several features of the problem, including exercise intolerance (1). In the early $20^{\text {th }}$ century, Sauerbruch's first patient was unable to work because of exercise-induced shortness of breath (2). Mark Ravitch commented a halfcentury later that patients could fool around playing basketball, but not play a whole game, or a few games of tennis, but not a set (3).

These observations by leaders in the field led to several efforts to measure pulmonary function. These were summarized by Shamberger in 2000 (4). Most of the studies were of small sample sizes, and the anatomic severity of the chest wall deformity was not recorded. The techniques available to measure pulmonary function gave very limited information, and pediatricians, family physicians, and internists were led to conclude that the condition had no effect on lung function.

Accordingly, efforts have been made to find out if patients with pectus excavatum have symptoms. A multicenter study, involving 11 North American medical centers, found that when asked, about $65 \%$ of patients complained of shortness of breath with exertion, and a similar number reported exercise intolerance (5). These same patients and their parents were asked by personnel not connected with their clinical care about these issues. The data showed overwhelming evidence of these complaints, both as reported by patients, and also separately by their parents (6). In the United States, the popularity of video games has led to a large group of young people who no longer perform exercise and therefore do not volunteer exercise intolerance as a symptom; rather, just as for other exercise-related phenomena, such as exercise-induced asthma, the clinician needs to specifically ask.

Between 1970 and 1987, a study of 152 pectus excavatum patients with an average age of 15 years was done without objective criteria for comparison purposes. This showed that preoperatively, the measured vital capacity was only $78 \%$ of predicted values, with a normal forced expiratory volume (FVC) 1/vital capacity (VC) ratio, indicating a restrictive defect. Unfortunately the open repair resulted in a decrease in total lung capacity (TLC), FEV1 and VC despite the fact that after the open pectus repair, the number of patients with decreased exercise tolerance or shortness of breath on exertion was reduced (7). Another similarly-powered study showed contrary findings (8).

Around the time of Nuss' first report in 1997, there was a realization that open operation could be complicated by "acquired Jeune's syndrome" (9). This postoperative complication occurred in very young children (aged 3 to 5 years) and involved failure of the chest wall to grow following overly extensive resection of costal cartilages. Teenage patients were left with a very small thoracic cage and attendant disability (10). Calcification of the costal cartilages, which regenerate following their removal, also occurred with the open approach (11). These complications suggested that the findings by Morshuis et al. of postoperative declines in pulmonary function tests (PFTs) were due to stiffness in the regenerated cartilages. There was immediate interest in pulmonary function after the Nuss procedure, since in that operation the cartilages are not removed, but only reshaped, thus maintaining flexibility.

As had been the case for the open operation, early reports involved small sample sizes. Borowitz et al. found that in 10 Nuss procedure patients in New York, there was no improvement but also no adverse effect on either static pulmonary function tests (PFT's) or on the ventilator response to exercise (12). This was important because it 


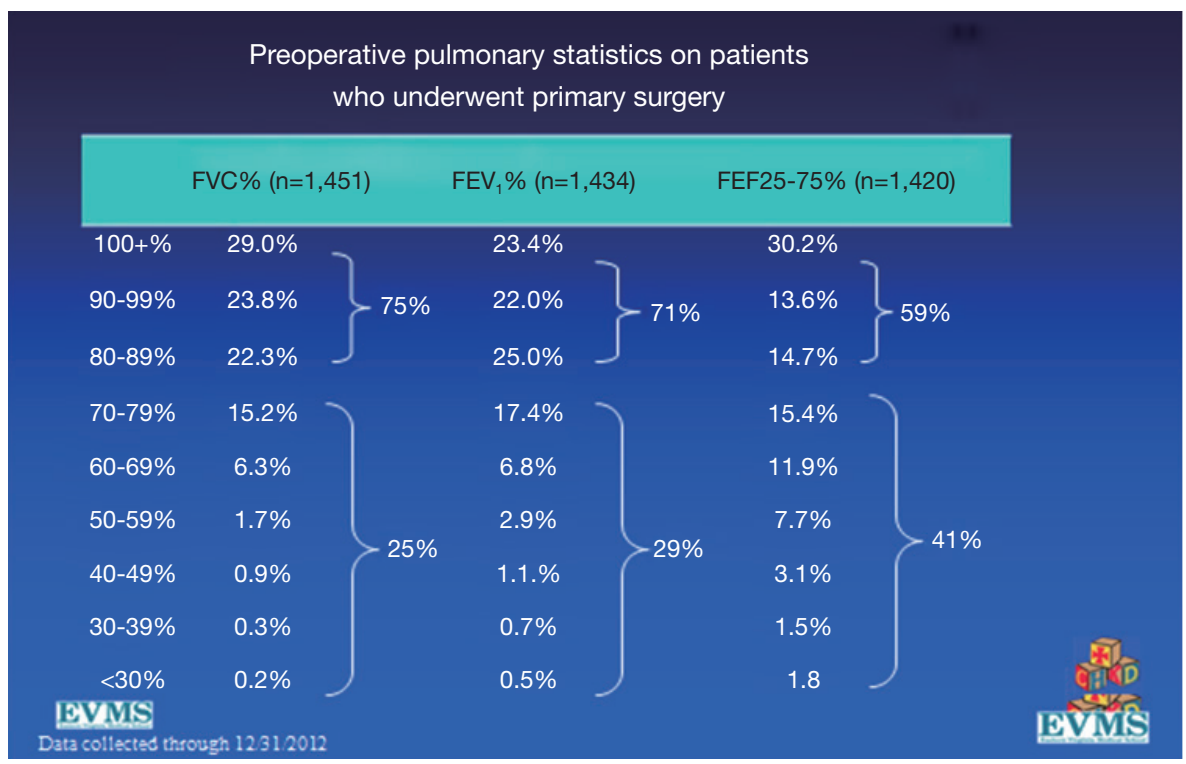

Figure 1 Note that a normally distributed population would have $50 \%$ of patients $100 \%+$, and $2.5 \%$ of patients less than $80 \%$ predicted. FVC, forced vital capacity; FEV1, forced expiratory volume; FEF, forced expiratory flow.

addressed concerns that the longer steel bar used in the Nuss procedure might restrict chest wall motion. However, there was no improvement of PFTs in those patients, either.

Our group from Norfolk, Virginia reported in 2005 on 408 Nuss operation patients and a subset of 45 patients seen following bar removal, and found different results. FVC improved from $85 \%$ of predicted values to $90 \%$; FEV 1 from $85 \%$ to $87 \%$; and FEF $25-75$ from $79 \%$ to $87 \%$ (13).

Subsequently, investigators in Basel, Switzerland reported 15 Nuss patients, finding that VC was $63.7 \%$ of predicted values and FEV1 $62.9 \%$ of predicted values preoperatively, (consistent with preoperative restriction) and improved by 38 and $40 \%$ respectively postop. TLC improved by $9.4 \%$. Patients with a pectus severity (Haller) index greater than 6 showed a greater effect (14). Contradicting these findings was a report from the Netherlands. Herein, a study of 203 patients found that at 6 months after bar insertion, the TLC, functional residual capacity (FRC), VC, FEV1, and mid-expiratory flow (MEF) 50 showed a significant increase. However, at 6 months after Nuss bar removal, in the 53 patients who returned for testing, none of the lung function variables showed any significant change compared to the preoperative values (15).

In an effort to get a broad sample from a large geographic area, a multicenter study of pectus excavatum and its treatment in North America was organized. In a group of 327 patients from 11 North American medical centers, FVC improved from $87.9 \%$ to $92.6 \%$, FEV1 from $86.6 \%$ to $90.1 \%$, and TLC from 94.3 to $100.2 \%$ of predicted values (16). Each of these gains was statistically significant. Patients with asthma or reactive airways disease were excluded from the study. We have been recording pre- and post-operative pulmonary function results on all patients since the early 1990s and have reviewed these studies in detail previously (6). We reported on a 21 year experience with PFT measurement, as shown in Figure 1, which illustrates a marked and statistically significant shift to the left in the PFTs (17). Our current data set, which now comprises more than 1,500 patients, including children and adults, shows no change in the previous pattern (Figures 1-6). There is a clear shift to lower FVC, FEV1, and FEF2575 in pectus patients, which improves after operation. These data are consistent with our clinical reports that only $65-70 \%$ of patients complain of exercise intolerance or shortness of breath with exertion. They also are consistent with our reported observation that not all patients improve with operative treatment. But taken as a whole, in large numbers, pectus patients do have a significant decrease in static pulmonary function pre-operatively, and that improves following the Nuss procedure.

\section{Discussion}

It is important to put these findings into context-PFTs 


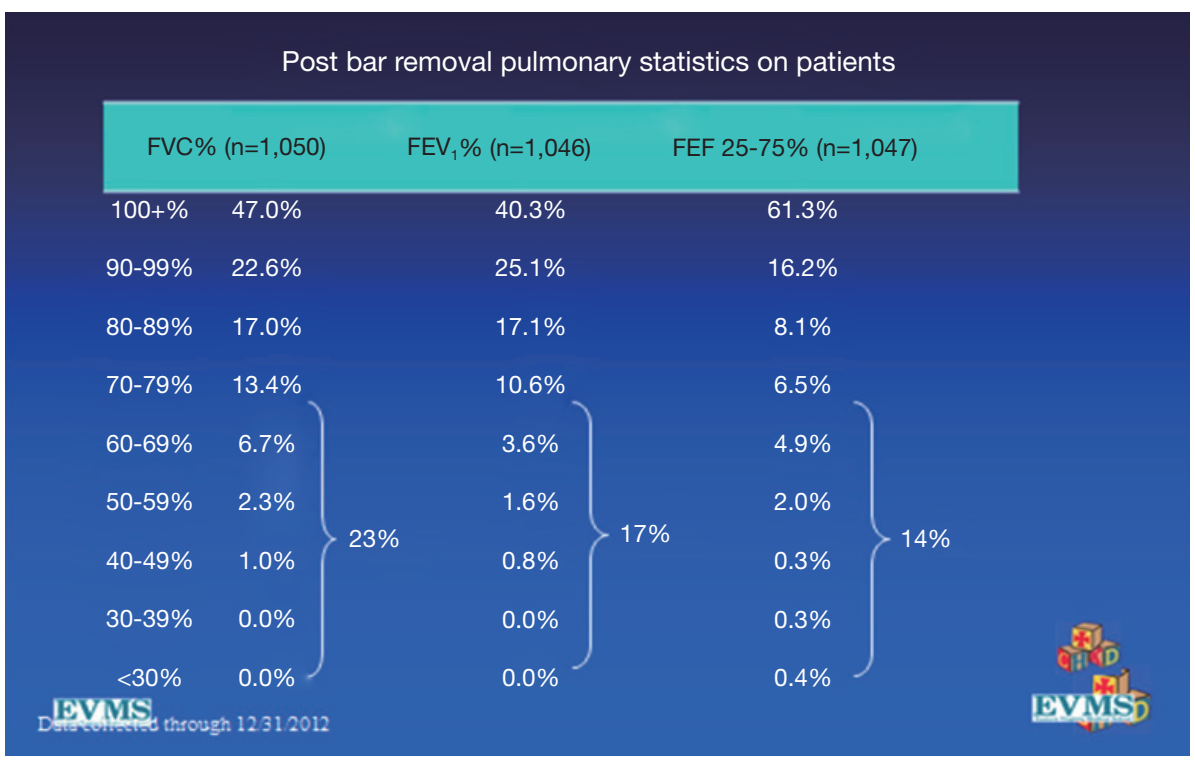

Figure 2 Note the large increase in patients in the 100\%+ group, and decreases in the < $80 \%$ predicted groups. FVC, forced vital capacity; FEV1, forced expiratory volume; FEF, forced expiratory flow.

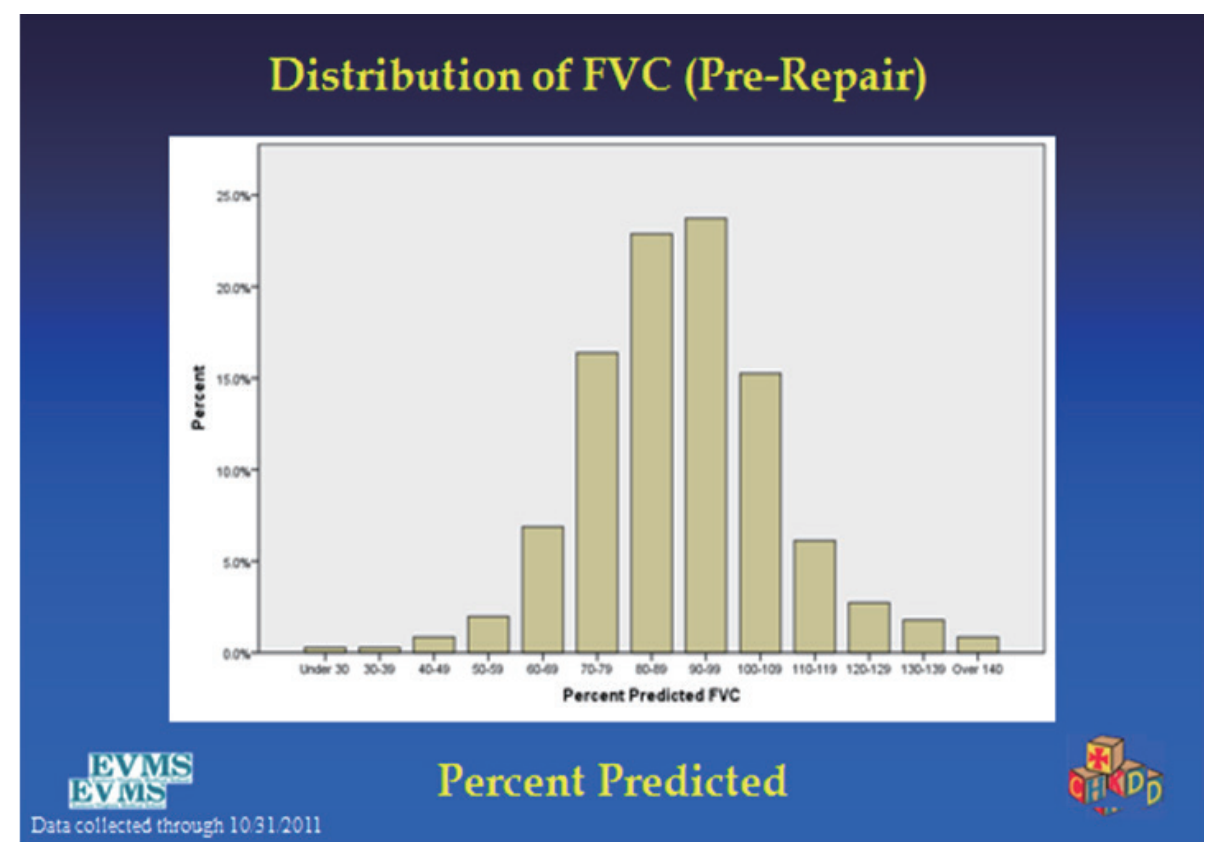

Figure 3 This distribution would be centered at 100\% if normally distributed. FVC, forced vital capacity.

are reported against findings in a normal population, and in that population, the values are distributed according to a Gaussian (bell-curve) distribution. For pulmonary function, $100 \%$ predicted is under the center of the bell curve, or at the average for the population. Two standard deviations below were defined as $80 \%$ predicted, and $120 \%$ was fixed at 2 standard deviations above average. Thus an improvement of $10 \%$ predicted, for example, is about one standard deviation. This degree is certainly clinically meaningful, even if not associated with symptoms. It has 


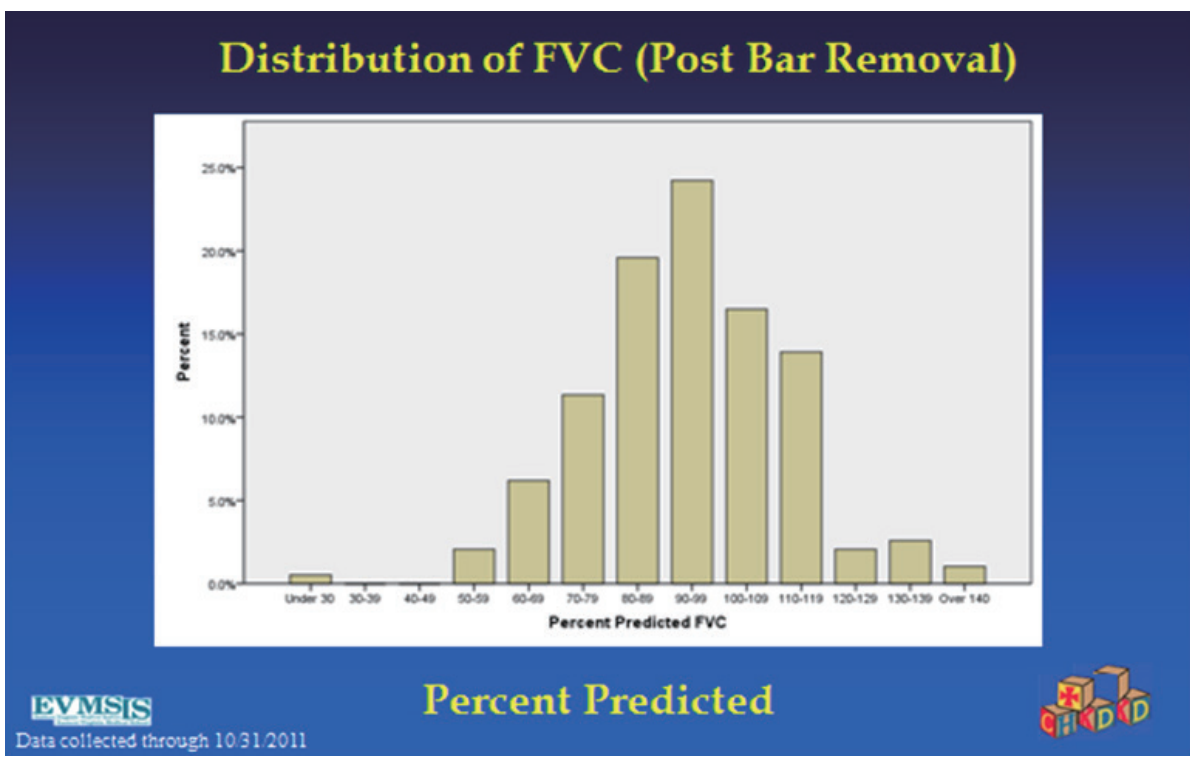

Figure 4 Note shift to the right. FVC, forced vital capacity.

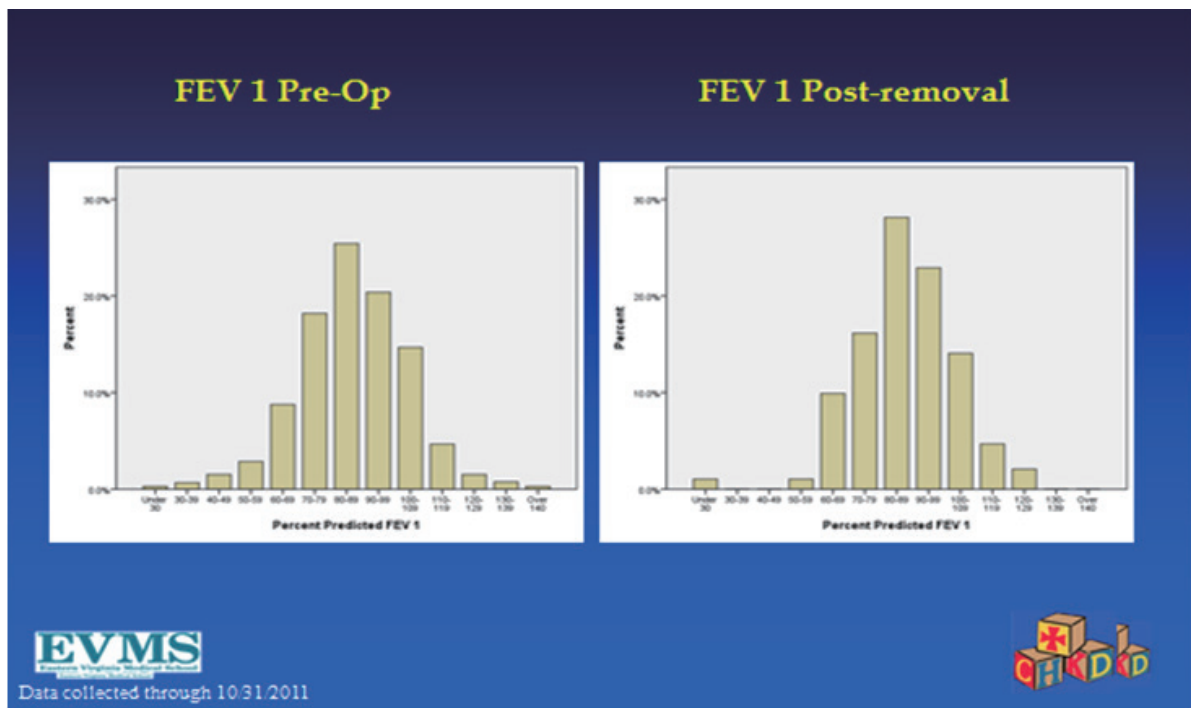

Figure 5 Note same phenomena as in FVC are recorded for FEV1. FVC, forced vital capacity; FEV1, forced expiratory volume.

been recognized that most otherwise healthy teenage pectus patients do not suffer from pulmonary parenchymal disease or airway disease. The chest wall disease itself does not affect the airways or pulmonary parenchyma except by mechanical compression. In Norfolk, which has a high prevalence of asthma and related disorders, asthma occurs at the same frequency in pectus patients as in the rest of the population (about $10 \%$ ). Importantly, in our previous published reports, patients with known asthma were excluded from PFT analysis. Any improvement in lung function after surgical repair of the chest wall must result from improvement in the mechanics of respiration, not improvement of obstruction to airflow at the airway.

Previous efforts to elucidate this mechanism have demonstrated the complexity of the anatomic bellows. The mechanism to draw air into the chest is simple: increasing the intrathoracic volume decreases the pressure below atmospheric pressure, and opening the airway to the 


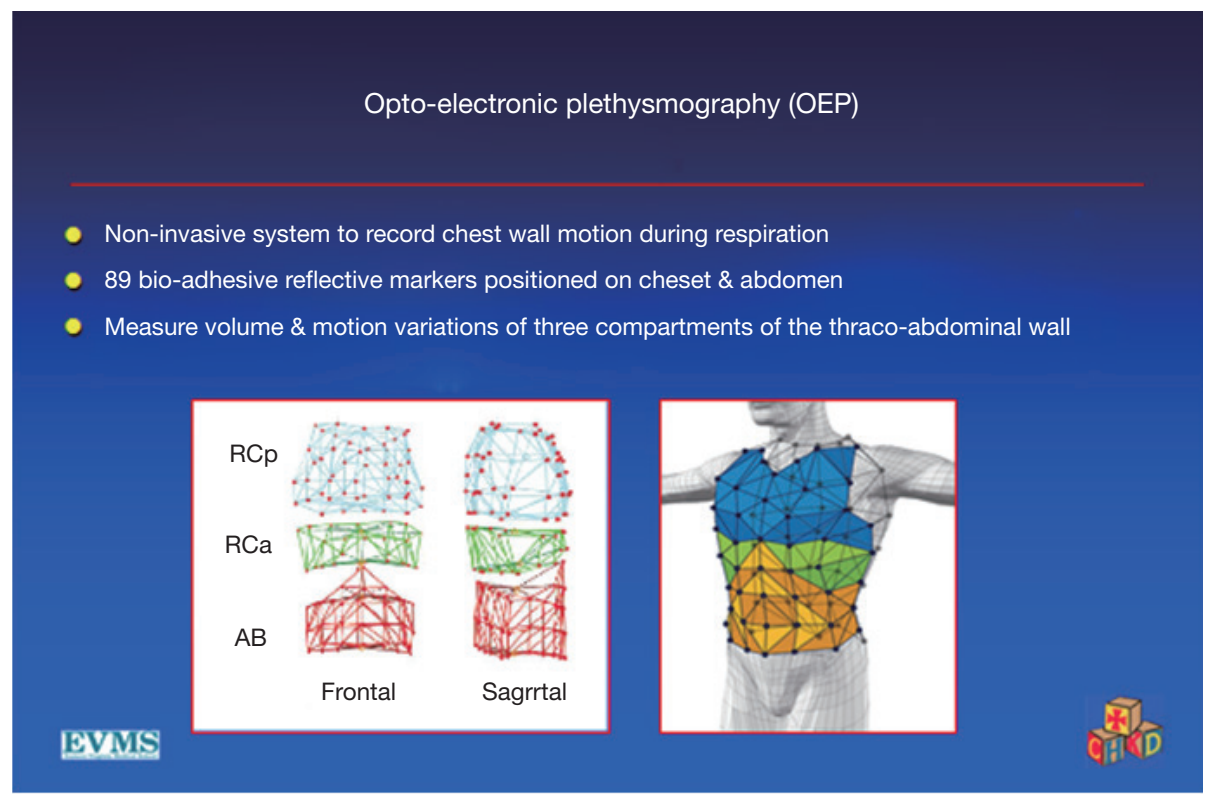

Figure 6 OEP divides chest into three horizontally arranged segments; but pectus depression commonly crosses these boundaries.

atmosphere causes air to flow from high to low pressure areas. However, understanding how this pressure decrease is achieved is not so simple. Pectus excavatum has been reported to occur with chronic upper airway obstruction in young children, and to be resolved by relief of the obstruction (18-20). Apparently, upper airway obstruction leads to marked increases in respiratory effort, which decreases the pressure. This does not seem to be the case in obstruction of the small airways, as noted in the preceding paragraph.

Diaphragmatic contraction contributes greatly to increasing the intrathoracic volume. It is the predominant factor at rest, in quiet tidal breathing. As the chest volume expands, diaphragmatic contraction and its effect on increasing the intrathoracic volume is not linear. The intercostal and scalene muscles contribute to increased intrathoracic volume only at higher volumes (during exercise), not in quiet breathing (21). To date, measurement of intrathoracic pressure requires a pressure gauge inside the body cavity, for example, in the esophagus. These are difficult investigations, both technically and for subject recruitment. In pectus excavatum patients, measurement of gastric pressure changes with respiration suggested that limitation of rib cage mobility is associated with increased abdominal pressure during inspiration, and that in patients with chest-wall strapping there were marked rises in endinspiratory abdominal pressure at rest, after exercise, and at total lung capacity. These rises were proportional to the degree of rib cage restriction (22). From these data, the authors concluded that chest wall mobility was not the cause of exercise intolerance in pectus excavatum.

Recently, however, motion capture technology has enabled evaluation of actual and direct movement of the chest wall (23). At least three centers have studied pectus patients using oculoelectronic plethysmography (OEP). After evaluation of seven pectus patients before and after operation, a group from the United Kingdom concluded that the $44 \%$ improvement in exercise capacity they measured at 6 months postoperatively was not due to improvement in chest wall motion. Similarly, a group from Italy, which studied 24 patients, concluded that that mild rib cage distortion rarely occurs in PE patients with mild restrictive defects $(24,25)$.

However, these investigators, including the inventors of the OEP device, chose to examine their data by dividing the chest into three vertically organized compartments: and these compartments could all be affected by chest wall deformity (Figure 7). It has been suggested that the rib cage in pectus excavatum fails to move up and out during inspiration (26). Normally, the sternum should move like the handle of an old-fashioned water pump, up and out with inspiration, and down and in with expiration. In performing OEP on 119 patients (64 pectus excavatum, 55 matched controls), we found that averaging all the points of the chest 


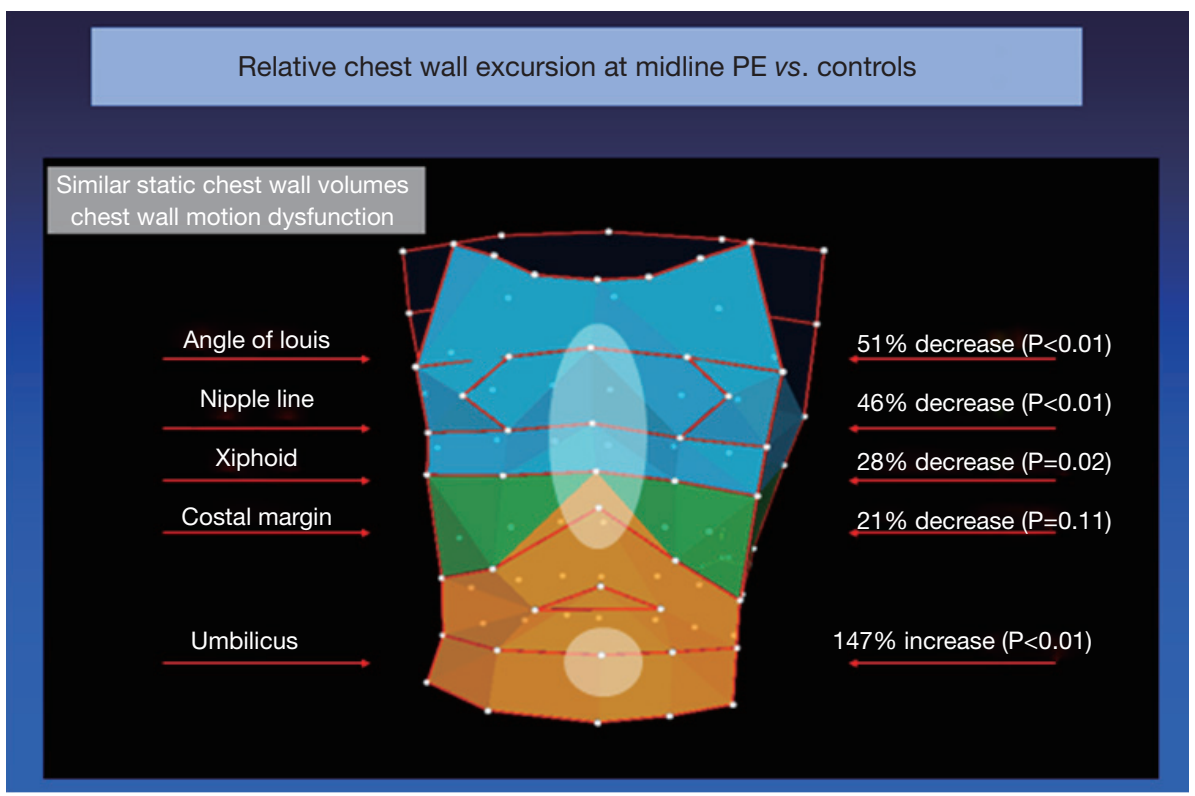

Figure 7 Decreased chest wall motion in 64 pectus patients vs. 55 matched controls. PE, pectus excavatum.

cage showed little abnormality when we first looked at our data. We then went back and watched the movement of the chest wall in patients, and could see that only the depression was fixed. Most of the chest wall moves very well in pectus excavatum.

Only the lower sternum and attached depressed costal cartilages do not move properly in the disease. In the Nuss procedure, only the depressed portion of the chest wall and its attached cartilages are moved to their normal location. OEP showed that the excavatum sternum was essentially fixed in position through the respiratory cycle, and that abdominal breathing was used to compensate, confirming what was suggested by the abdominal pressure studies $(27,28)$. There is a regional chest wall motion dysfunction (Figures 7,8).

Following the Nuss procedure, chest wall motion, including at the site of the former depression, was indistinguishable from controls, even with the bar in place. There were marked increases in thoracic compartment volumes, including a gain of more than a liter in total chest wall volume (28)!

A comparison of PFTs from patients in the multicenter study showed that those with higher Haller index were more likely to have diminished PFT's than those with lower. Patients with a Haller index greater than 7 (Normal index, 2.2, severe pectus excavatum $>3.25$ ) were four times more likely to have a restrictive pattern on PFT's than those with an index $<7$ (restrictive pattern being defined as FVC $<80 \%$ predicted, with a normal ratio of FEV1 to FVC) (29). This is consistent with the OEP observations, since patients with deeper depressions have greater volume fixed incursion into the bony thorax.

Increased understanding of the pathophysiology of pectus excavatum should move on to discussions of mechanisms of dysfunction. In large series, only about $40 \%$ of patients have a family history (18), demonstrating that in the majority of patients there is either a de novo problem with the structure of the chest or no such problem. Indeed, the success of surgical correction, either by open or Nuss procedure, argues against an intrinsic problem of the chest wall itself: for if the chest wall were structurally deficient, recurrence should follow repair frequently. Yet it does not: large series of patients from around the world cite a $1-2 \%$ recurrence rate after Nuss procedure, in which the chest wall itself is not resected, but only remodeled.

Since patients with pectus excavatum are generally healthy, and the incidence of asthma and related bronchospastic disorders is the same as the general population, the airflow in and out of the chest is only different in pectus patients in the effectiveness of the bellows action of the chest. OEP has demonstrated that the lower portion of the sternum does not move well in patients with pectus excavatum, and that the movement of the 


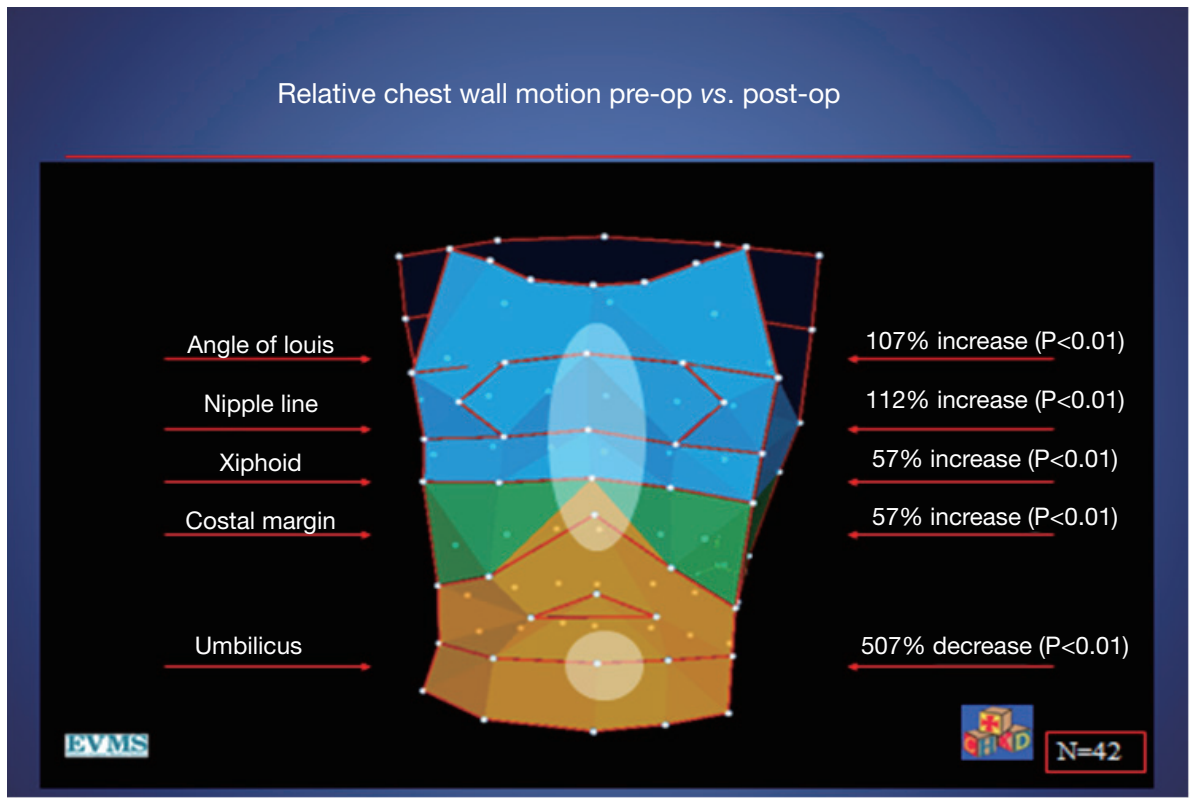

Figure 8 Restoration of chest wall motion in 42 patients at 6 months postoperatively.

affected area is normal postoperatively. Further, OEP shows that the abdominal contribution to respiration is markedly decreased after surgical correction: so that patients with pectus excavatum use abdominal breathing to compensate for less effective motion of the lower sternum. This is less efficient during exercise, and explains the lack of endurance cited by two-thirds of patients in our data. After surgical correction, breathing is more efficient.

Shortness of breath with exertion and easy fatigability were cited by about two-thirds of patients presenting to us. But what about the other one-third? We have shown that the likelihood of restrictive findings on PFTs is related to the severity of the pectus by Haller index. Given the deficiencies of the Haller index, future efforts to understand the problem should use other methods, such as the pectus correction index, or external chest wall scanning.

Physicians will need to stop viewing pectus excavatum as a binary phenomenon: either present or absent. We have relearned in the last 20 years that the overall shape of the thorax is markedly different in different people: a $3 \mathrm{~cm}$ depression in a barrel-chested individual is very different to a $3 \mathrm{~cm}$ depression in a person with a very shallow anteriorposterior depth. Similarly, efforts to study respiratory excursion need to begin to take into account the $20 \%$ of patients who have concurrent scoliosis and pectus deformities. Both problems are known to have respiratory effects.

Recently, the limitation of the Haller index in demonstrating severity in barrel chested individuals has been noted, and an alternative index proposed (the correction index) (30). This index better addresses the phenomena of "barrel" and "pancake" shaped chests with deformity than the Haller index.

The cardiac effects of pectus repair, through relief of cardiac compression or restricted chest expansion, may be the predominant reason why patients feel that they can exercise better and is discussed elsewhere in this journal. However, the heart and lung system are a coupled system, and improvement of either will likely favorably affect the other. Multiple studies of exercise PFTs have shown that the oxygen pulse, which is a surrogate for stroke volume, is increased in pectus excavatum (31-33). Morshuis and colleagues reported a significant increase in maximal oxygen uptake and oxygen pulse (33). In the multicenter study of pectus excavatum, $\mathrm{VO}_{2}$ max increased by $10 \%$ and oxygen pulse by $20 \%$ in the 20 patients who completed both pre- and postoperative testing. The largest number of patients were treated by the open or Ravitch operation (16). Recently, cardiac MRI and transesophageal echocardiography have has been advocated as better methods to understand the cardiac effects of the problem (34).

The generalizability of this report is limited by its design 
as a retrospective, single center review of prospectively acquired data.

\section{Conclusions}

Recent pulmonary function studies of large groups of pectus excavatum patients have provided more clarity to the question of whether pectus excavatum has physiologic effects. Efforts to understand chest wall deformity need to utilize any newly available methods to identify the mechanisms producing limitation of cardiopulmonary function in this disease. Tying these findings to the genetic phenomenon, given that pectus excavatum disproportionately affects one sex (75-80\% of patients worldwide are male), is inherited in a considerable proportion of cases (approximately 40\%), and is associated with connective tissue disorder (Marfan or Ehlers Danlos syndromes) about $15-20 \%$ of patients, should be a focus for future studies.

\section{Acknowledgements}

None.

\section{Footnote}

Conflicts of Interest: All three authors are consultants for Zimmer Biomet.

\section{References}

1. Bauhinus J, Schenck von Grafenberg J. Observationum medicarum, rarum, novarum, admirabilium, et monstrosarum, liber secundus. Departibus vitalibus, thorace contentis. Observation 1594;264:516.

2. Sauerbruch F. Operative Beseitigung der Angeborenen Trichterbrust. Deutsche Zeitschr f. Chir 1931;234:760.

3. Ravitch MM. The chest wall. In: Pediatric surgery. 4th edition. Welch KJ et al. (Eds.). Chicago: Year Book Medical Pub 1986:568-78.

4. Shamberger RC. Cardiopulmonary effects of anterior chest wall deformities. Chest Surg Clin N Am 2000;10:245-52, v-vi.

5. Kelly RE Jr, Shamberger RC, Mellins RB, et al. Prospective multicenter study of surgical correction of pectus excavatum: design, perioperative complications, pain, and baseline pulmonary function facilitated by internet-based data collection. J Am Coll Surg
2007;205:205-16.

6. Kelly RE Jr, Cash TF, Shamberger RC, et al. Surgical repair of pectus excavatum markedly improves body image and perceived ability for physical activity: multicenter study. Pediatrics 2008;122:1218-22.

7. Morshuis WJ, Folgering HT, Barentsz JO, et al. Exercise cardiorespiratory function before and one year after operation for pectus excavatum. J Thorac Cardiovasc Surg 1994;107:1403-9.

8. Haller JA Jr, Loughlin GM. Cardiorespiratory function is significantly improved following corrective surgery for severe pectus excavatum. Proposed treatment guidelines. J Cardiovasc Surg (Torino) 2000;41:125-30.

9. Robicsek F, Fokin AA. How not to do it: restrictive thoracic dystrophy after pectus excavatum repair. Interact Cardiovasc Thorac Surg 2004;3:566-8.

10. Haller JA Jr, Colombani PM, Humphries CT, et al. Chest wall constriction after too extensive and too early operations for pectus excavatum. Ann Thorac Surg 1996;61:1618-24; discussion 1625.

11. Chang PY, Lai JY, Chen JC, et al. Long-term changes in bone and cartilage after Ravitch's thoracoplasty: findings from multislice computed tomography with 3-dimensional reconstruction. J Pediatr Surg 2006;41:1947-50.

12. Borowitz D, Cerny F, Zallen G, et al. Pulmonary function and exercise response in patients with pectus excavatum after Nuss repair. J Pediatr Surg 2003;38:544-7.

13. Lawson ML, Mellins RB, Tabangin M, et al. Impact of pectus excavatum on pulmonary function before and after repair with the Nuss procedure. J Pediatr Surg 2005;40:174-80.

14. Kubiak R, Habelt S, Hammer J, et al. Pulmonary function following completion of Minimally Invasive Repair for Pectus Excavatum (MIRPE). Eur J Pediatr Surg 2007;17:255-60.

15. Aronson DC, Bosgraaf RP, Merz EM, et al. Lung function after the minimal invasive pectus excavatum repair (Nuss procedure). World J Surg 2007;31:1518-22.

16. Kelly RE Jr, Mellins RB, Shamberger RC, et al. Multicenter study of pectus excavatum, final report: complications, static/exercise pulmonary function, and anatomic outcomes. J Am Coll Surg 2013;217:1080-9.

17. Kelly RE, Goretsky MJ, Obermeyer R, et al. Twentyone years of experience with minimally invasive repair of pectus excavatum by the Nuss procedure in 1215 patients. Ann Surg 2010;252:1072-81.

18. Fan L, Murphy S. Pectus excavatum from chronic upper airway obstruction. Am J Dis Child 1981;135:550-2. 
19. Olsen KD, Kern EB, O'Connell EJ. Pectus excavatum: resolution after surgical removal of upper airway obstruction. Laryngoscope 1980;90:832-7.

20. Lane RW, Weider DJ, Steinem C, et al. Laryngomalacia. A review and case report of surgical treatment with resolution of pectus excavatum. Arch Otolaryngol 1984;110:546-51.

21. Decramer M, Jiang TX, Demedts $M$. Effects of acute hyperinflation on chest wall mechanics in dogs. J Appl Physiol (1985) 1987;63:1493-8.

22. Mead J, Sly P, Le Souef P, et al. Rib cage mobility in pectus excavatum. Am Rev Respir Dis 1985;132:1223-8.

23. Seddon P. Options for assessing and measuring chest wall motion. Paediatr Respir Rev 2015;16:3-10.

24. Binazzi B, Innocenti Bruni G, Coli C, et al. Chest wall kinematics in young subjects with Pectus excavatum. Respir Physiol Neurobiol 2012;180:211-7.

25. Binazzi B, Innocenti Bruni G, Gigliotti F, et al. Effects of the Nuss procedure on chest wall kinematics in adolescents with pectus excavatum. Respir Physiol Neurobiol 2012;183:122-7.

26. Wohl ME, Stark A, Stokes DC. Thoracic disorders in childhood. In: Roussos C (Ed.), The Thorax Part C New York: Marcel Dekker Inc. 1995:2035-69.

27. Redlinger RE Jr, Kelly RE, Nuss D, et al. Regional chest wall motion dysfunction in patients with pectus excavatum demonstrated via optoelectronic plethysmography. J

Cite this article as: Kelly RE Jr, Obermeyer RJ, Nuss D. Diminished pulmonary function in pectus excavatum: from denying the problem to finding the mechanism. Ann Cardiothorac Surg 2016;5(5):466-475. doi: 10.21037/ acs.2016.09.09
Pediatr Surg 2011;46:1172-6.

28. Redlinger RE Jr, Wootton A, Kelly RE, et al.

Optoelectronic plethysmography demonstrates abrogation of regional chest wall motion dysfunction in patients with pectus excavatum after Nuss repair. J Pediatr Surg 2012;47:160-4.

29. Lawson ML, Mellins RB, Paulson JF, et al. Increasing severity of pectus excavatum is associated with reduced pulmonary function. J Pediatr 2011;159:256-61.

30. St Peter SD, Juang D, Garey CL, et al. A novel measure for pectus excavatum: the correction index. J Pediatr Surg 2011;46:2270-3.

31. Sigalet DL, Montgomery M, Harder J. Cardiopulmonary effects of closed repair of pectus excavatum. J Pediatr Surg 2003;38:380-5; discussion 380-5.

32. Morshuis W, Folgering H, Barentsz J, et al. Pulmonary function before surgery for pectus excavatum and at longterm follow-up. Chest 1994;105:1646-52.

33. Maagaard M, Tang M, Ringgaard S, et al. Normalized cardiopulmonary exercise function in patients with pectus excavatum three years after operation. Ann Thorac Surg 2013;96:272-8.

34. Humphries CM, Anderson JL, Flores JH, et al. Cardiac magnetic resonance imaging for perioperative evaluation of sternal eversion for pectus excavatum. Eur J Cardiothorac Surg 2013;43:1110-3. 